\title{
Acute Kidney Injury in Severe Preeclamptic Patients Admitted to Intensive Care Unit: Epidemiology and Role of Serum Neutrophil Gelatinase-associated Lipocalcin
}

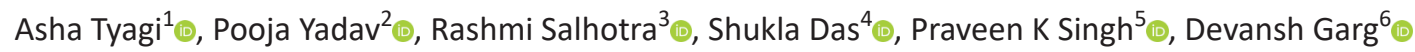

\begin{abstract}
Background: Patients with preeclampsia admitted to the intensive care unit (ICU) may have risk factors for acute kidney injury (AKI). Although the use of neutrophil gelatinase-associated lipocalcin (NGAL) to predict AKI is previously validated, we could locate only scanty data regarding the epidemiology of AKI and role of NGAL in preeclamptic patients admitted to ICU.

Methods: Patients with preeclampsia admitted to our ICU were included. The incidence and severity of AKI during the entire ICU stay were assessed using kidney disease improving global outcomes criteria, while the a priori risk factors and serum NGAL were also evaluated.

Results: A total of 52 preeclamptic patients admitted to ICU were included, among whom the majority had eclampsia (75\%). AKI developed in 25 (48.1\%) patients with stages 1, 2, and 3 in 56,36 , and $8 \%$, respectively. The incidence of sepsis ( 16 vs $0 \%)$, shock (40 vs $7.4 \%)$, and anemia ( 84 vs $59.3 \%$ ) was significantly greater in patients with AKI $(p<0.05)$. ICU mortality ( 28 vs $3.7 \%)$, duration of ICU, and hospital stay were significantly higher in patients who developed AKI $(p<0.05)$. There was no association of serum NGAL [274 $(240-335) \mathrm{ng} / \mathrm{mL}]$ with AKI or the mortality $(p=0.725,0.861)$; there was, however, a significant discriminatory value for eclampsia $[p=0.019$; area under curve $=0.736$ (95\% confidence interval: 0.569-0.904)].

Conclusions: Although AKI is common among patients with preeclampsia admitted to ICU, serum NGAL does not predict its occurrence.

Keywords: Acute kidney injury, Intensive care unit, Neutrophil gelatinase-associated lipocalcin, Preeclampsia, Pregnancy.

Indian Journal of Critical Care Medicine (2021): 10.5005/jp-journals-10071-23966
\end{abstract}

\section{INTRODUCTION}

Preeclampsia is a commonly encountered hypertensive disorder of pregnancy, in both developed as well as developing countries (incidence of $3-4 \%$ and up to $15 \%$, respectively). ${ }^{1-3}$ The disease continues to be associated with maternal as well as fetal mortality.

Preeclampsia has multisystemic manifestations, with renal affliction being frequently encountered. ${ }^{3,4}$ Both glomerular as well as tubular renal involvement are known to occur. ${ }^{4-6}$ Preeclampsia can also blunt the pregnancy-induced physiological increase in glomerular filtration rate (GFR), or even decrease it. ${ }^{7}$ Lastly, there may be a prerenal contribution as well, ${ }^{4}$ following hypoperfusion and blood loss. Due to all of the above reasons, preeclampsia is known to be a risk of development of acute kidney injury (AKI)., ${ }^{3,8}$

The presence of AKI increases morbidity, mortality, as well as the incidence of long-term renal failure. ${ }^{8}$ Previous incidences of AKI in mixed-population patients admitted to the intensive care unit (ICU) are up to $10-30 \%$ and $61 \%$ in an obstetric subgroup. ${ }^{9}$ Despite the increased risk of AKI in preeclampsia, there are, however, only limited data on the association and its implications. The limited data are derived from patients with preeclampsia who were hospitalized, but not admitted to ICU. The risk factors may be more prevalent and more numerous, and hence the incidence of AKI is greater in preeclamptics who need ICU care.

Also, the conventionally recommended diagnosis of AKI by an increase in serum creatinine and/or fall in urine output has its own limitations. ${ }^{8}$ The increase in serum creatinine occurs much later than the structural renal injury and remains a nonspecific marker as well. ${ }^{8}$ Various urinary and serum biomarkers have been evaluated with the aim of early detection of AKI and an 1-3,6 Department of Anaesthesiology and Critical Care, University
College of Medical Sciences and GTB Hospital, Delhi, India
${ }^{4,5}$ Department of Microbiology, University College of Medical Sciences
and GTB Hospital, Delhi, India

Corresponding Author: Asha Tyagi, Department of Anesthesiology and Critical Care, University College of Medical Sciences and GTB Hospital, Delhi, India, Phone: +919818606404, e-mail: drashatyagi@ gmail.com

How to cite this article: Tyagi A, Yadav P, Salhotra R, Das S, Singh PK, Garg D. Acute Kidney Injury in Severe Preeclamptic Patients Admitted to Intensive Care Unit: Epidemiology and Role of Serum Neutrophil Gelatinase-associated Lipocalcin. Indian J Crit Care Med 2021;25(9):1013-1019.

Source of support: Nil

Conflict of interest: None

associated improvement in the patient outcome. Neutrophil gelatinase-associated lipocalcin (NGAL) levels in urine or serum have been validated as a biomarker for detecting AKI early on since it is released within 3-6 hours of renal injury. Interestingly in preeclamptic patients, several studies have evaluated the role of serum NGAL to predict the onset and severity of preeclampsia itself. Despite being a known marker for AKI as well as preeclampsia individually, only one previous study analyzed the utility of serum NGAL as a predictor of AKI in preeclamptic patients. ${ }^{10}$

Against this background, the present prospective cohort study aimed to evaluate the epidemiology of AKI and also the role of 
serum NGAL in predicting the occurrence of AKI and mortality for patients with preeclampsia who were admitted to the ICU.

\section{Materials and Methods}

This prospective observational cohort study was undertaken after obtaining clearance from the Institutional Ethical CommitteeHuman Research, University College of Medical Sciences, in a meeting held on October 13,2018. The study is prospectively registered with the Clinical Trial Registry of India (CTRI/2018/12/016617). For all the patients, written informed consent from self/next of kin was taken prior to inclusion in the study.

The primary objective was to evaluate the epidemiology of AKI in these patients. The secondary objective was to evaluate the role of serum NGAL estimated at the time of ICU admission for prediction of AKI, as well as mortality.

All patients with preeclampsia (pregnant or within 6 weeks postdelivery), who required admission to the ICU and were expected to have a stay of 24 hours at least, were included. Those not requiring insertion of a Foley's catheter, having a history of renal dysfunction or needing renal replacement therapy prior to present hospitalization, or with renal transplant were excluded from the study.

Diagnosis of preeclampsia was made by attending obstetrician/ confirmed by intensivist as per previous guidelines ${ }^{3}$ i.e., raised blood pressure (systolic more than or equal to $140 \mathrm{~mm} \mathrm{Hg}$ or diastolic more than or equal to $90 \mathrm{~mm} \mathrm{Hg}$ on two occasions at least 4 hours apart) along with proteinuria (more than or equal to $300 \mathrm{mg} / 24$ hours or protein/creatinine ratio more than or equal to 0.3 or dipstick $1+$ ) after 20 weeks of gestation, or any of the following features: thrombocytopenia (platelets $<100,000 / \mu \mathrm{L}$ ), impaired liver function, new development of renal insufficiency, pulmonary edema, new-onset cerebral, or visual disturbances. In the case of more than one admission to ICU for the same patient, only the first one was considered in order to avoid bias. If the patient was readmitted within 24 hours after initial discharge, it was considered in continuation of the initial admission.

\section{Operational Definitions}

\section{Acute Kidney Injury}

The occurrence of AKI in the ICU was defined as per the kidney disease improving global outcomes (KDIGO) clinical practice guidelines for $A K I^{8}$ i.e., an increase in serum creatinine $\geq 0.3 \mathrm{mg} / \mathrm{dL}$ within 48 hours, or increase in serum creatinine to $>1.5$ times baseline, which was known or presumed to have occurred within the prior 7 days, or urine volume $<0.5 \mathrm{~mL} / \mathrm{kg} / \mathrm{hour}$ for 6 hours. Only when oliguria did not respond to the intravenous fluid challenge was it considered as indicative of AKI.

The various grades of AKI severity included stages 1 (mild), 2 (moderate), and 3 (severe) depending upon the extent of derangement in serum creatinine or urine output. ${ }^{8}$

\section{Risk Factors for Aki}

Bleeding originating from or into the genital tract occurring from 24 weeks of pregnancy onward till prior to the birth of the baby represented antepartum hemorrhage. Placenta previa and placental abruption were cited as the most important causes of antepartum hemorrhage.

Postpartum hemorrhage was defined as loss of blood of more than $500 \mathrm{~mL}$ after vaginal delivery, $1000 \mathrm{~mL}$ following cesarean section, or $1500 \mathrm{~mL}$ after hysterectomy.
Hemolysis, elevated liver enzymes, and low platelet (HELLP) syndrome was diagnosed by the presence of hemolysis (serum bilirubin $>1.2 \mathrm{mg} / \mathrm{dL}$ or lactate dehydrogenase $>600 \mathrm{U} / \mathrm{L}$ ), along with raised aspartate aminotransferase $(\geq 70 \mathrm{U} / \mathrm{L})$ and low platelets $(<100,000 / \mu \mathrm{L})$.

Puerperal sepsis was defined as an infection of the genital tract occurring at any time from onset of rupture of membranes or labor to postpartum period accompanied with any two or more out of pelvic pain, fever (temperature $\geq 38.5^{\circ} \mathrm{C}$ ), and abnormal vaginal discharge i.e., presence of pus, abnormal smell/foul odor of discharge, or delay in the reduction of the size of the uterus (involution).

Disseminated intravascular coagulopathy and acute fatty liver were to be diagnosed as per clinical presentation along with laboratory tests if required.

A fall in the systolic blood pressure to $<90 \mathrm{~mm} \mathrm{Hg}$ (or a drop $>40 \mathrm{~mm} \mathrm{Hg}$ ), or a mean arterial pressure less than $65 \mathrm{mmHg}$ depicted shock was recorded.

Anemia was defined as hemoglobin less than $11 \mathrm{gm} / \mathrm{dL}$.

\section{Methodology}

For diagnosis and severity grading of AKI during the ICU stay, the serum creatinine was assessed at the time of ICU admission on the first day and then daily for all subsequent days of ICU stay; while urine output was monitored hourly during the ICU stay.

For diagnosing AKI using serum creatinine, baseline value was defined as the one obtained prior to ICU admission, but during current illness. In case the preadmission value was not available, it was estimated using the modification of diet in renal disease equation. The lower bound of normal GFR i.e., $75 \mathrm{~mL} /$ minute per $1.73 \mathrm{~m}^{2}$ was considered herein [GFR $=\exp [5.228-1.154 \times \mathrm{ln}$ (S.Cr.)] $\times \ln$ (age) $-(0.299$ if female $)+(0.192$ if black $)]$.

The management of AKI was as per existing clinical practice. The need for renal replacement therapy was decided by the nephrologist.

Evaluation for various a priori risk factors for AKI, as defined above, was done during the entire ICU stay or at the time of admission, as appropriate.

The outcome of a patient (survival or death) in ICU and hospital was recorded.

\section{Serum Biomarker Assay and Prediction of Outcome including Early AKI}

Blood sample (2 $\mathrm{mL}$ venous blood) was collected for NGAL determination at the earliest possible time of ICU admission. This was centrifuged and then kept at the temperature of $-80^{\circ} \mathrm{C}$, until assayed by the method of enzyme-linked immunosorbent assay kit as per manufacturer instructions (Biovender-laboratorni med, Czech Republic). The sensitivity for detection was $0.02 \mathrm{ng} / \mathrm{mL}$. A total of 5 of 52 samples (all from patients with HELLP syndrome) showed evidence of gross hemolysis upon serum separation and were hence not assessed for NGAL levels as per manufacturer instructions.

\section{Other Observations}

Demographic and obstetric characteristics pertaining to critically ill preeclamptic patients were noted, including receiving of antenatal care, parity, period of gestation (weeks), place of delivery, source of ICU admission, duration of illness prior to hospitalization, mode of fetal delivery, use of a diuretic, whether referred from another hospital, presence of comorbidities, need for mechanical ventilation, and duration of ICU stay. 
The severity of preeclampsia was recorded (preeclampsia, severe preeclampsia, or eclampsia).

The study period ended with the patient's ICU stay, except for the extended observation of inhospital mortality.

\section{Sample Size}

In an earlier study evaluating the incidence of AKI in critically ill obstetric patients, $47 \%$ of the preeclamptic subset was seen to develop AKI. ${ }^{9}$ Considering this earlier incidence, 48 patients would be required to detect $A K I$ at $a$ value of $5 \%$ and relative marginal error of $30 \%$. To accommodate dropouts or missing data a higher number of 52 patients were enrolled.

\section{Statistical Analysis}

Descriptive analysis using mean [standard deviation (SD)] or median [interquartile range (IQR)] or number (percentages) was performed for normally distributed quantitative data, nonnormally distributed data, and categorical values, respectively. Comparison of characteristics and risk factors between patients with and without AKI as well as ICU survivors vs non-survivors was made using $t$-test or Mann-Whitney $U$ test as appropriate. In addition, a univariate regression analysis was performed for evaluating predictive value of various risk factors for AKI. For analyzing the role of serum NGAL to predict AKI, mortality, and eclampsia, receiver-operating characteristics $(R O C)$ analysis was conducted.

\section{Results}

The total number of patients admitted to the ICU during the time of data collection was 554 (from February 2019 to February 2020). Of these, 163 were obstetric patients with 56 suffering from preeclampsia. From among the 56 preeclamptics, a convenience sample of 52 was included.
Baseline demographic characteristics and obstetric details of the included patients are depicted in Table 1. All the patients had a fetal delivery in our institution.

Approximately $23.1 \%$ of the patients (12/52) had comorbidity, including hypothyroidism $(n=7)$, severe anemia $(n=5)$, coexistent chronic hypertension $(n=1)$, or pulmonary thromboembolism $(n=1)$. More than 1 comorbidity was present in some patients.

All but one patient $(98.1 \%)$ required mechanical ventilation during ICU stay.

\section{Epidemiology of AKI}

AKI was seen in $48.1 \%$ ( 25 of 52 patients). Of these 25 patients, stage 1, 2, and 3 of severity was seen in 14 (56\%), 9 (36\%), and 2 (8\%) patients, respectively.

Of the total 52 patients, $29(55.8 \%)$ had a serum creatinine of $>1 \mathrm{mg} / \mathrm{dL}$ during the ICU stay.

Among patients with $\mathrm{AKI}, \mathrm{ICU}$ mortality showed a variation with severity: [stage $1: 4$ of $14(28.6 \%)$; stage $2: 1$ of $9(11.1 \%)$; and stage 3 : 2 of $2(100 \%)](p=0.001)$. There was no death of any patient after discharge from the ICU, making the inhospital mortality the same as ICU mortality ( 8 of $/ 52=15.3 \%)$.

Sepsis ( $16 \%$ vs $0 \%)$, shock ( $40 \%$ vs $7.4 \%)$, and anemia ( $84 \%$ vs $59.3 \%$ ) had a significantly greater incidence in patients with $\mathrm{AKI}$ as compared to those without ( $p<0.05$; Table 2 ).

Additionally, the severity of preeclampsia, in terms of progression to eclampsia, the occurrence of pulmonary edema, and the magnitude of proteinuria were also compared and noted to be statistically similar between patients with or without AKI $(p>0.05$; Table 2). Herein, for comparison of pulmonary edema, two patients suffering from cardiomyopathy along with were excluded.

From among all the above risk factors for AKI, upon univariate regression analysis, only shock remained a significant predictor $[p=0.012$; odds ratio (OR) $[95 \%$ confidence interval $(\mathrm{Cl})]=8.3(1.6-43.2)]$.

Table 1: Demographic characteristics and obstetric details $(n=52)$

\begin{tabular}{lc}
\hline Characteristic & Value \\
\hline Age (years) & $26.3(5.6)$ \\
Parity & $0(0-2)$ \\
Primigravida & $27(51.9 \%)$ \\
Pregnant at the time of ICU admission & $5(9.6 \%)$ \\
Period of gestation (weeks) & $34.8(3.9)$ \\
Antenatal care received & $9(17.3 \%)$ \\
Mild preeclampsia:severe preeclampsia:eclampsia & $0(0 \%): 13(25 \%): 39(75 \%)$ \\
Pulmonary edema due to preeclampsia & $7(13.4 \%)$ \\
HELLP syndrome & $12(23.1 \%)$ \\
Duration of illness prior to hospitalization (days) & $0.06(0.2)$ \\
& $0(0-0)$ \\
Duration of hospitalization prior to ICU admission (days) & $1(0-1)$ \\
Duration of ICU stay (days) & $1(1-2)$ \\
& $(1-19)$ \\
Duration of hospital stay (days) & $12(8-19)$ \\
Source of ICU admission (emergency OT:labor room) & $(1-43)$ \\
Mode of delivery (vaginal:cesarean:hysterotomy) & $47(90.4 \%): 5(9.6 \%)$ \\
Surgical intervention (cesarean:laparotomy:hysterotomy) & $5(9.6 \%): 45(86.5 \%): 2(3.8 \%)$ \\
Referred from other hospitals & $45(86.5 \%): 0(0 \%): 2(3.8 \%)$ \\
\hline
\end{tabular}

Values are mean (SD), median (IQR), or number of patients (\%); OT, operation theater; HELLP, hemolysis, elevated liver enzymes, and low platelets 
AKI in Critically Ill Preeclamptics

Table 2: Comparison of risk factors between patients with and without AKI

\begin{tabular}{lccc}
\hline Characteristic & $\begin{array}{c}A K I \\
(n=25)\end{array}$ & $\begin{array}{c}\text { Non-AKI } \\
(n=27)\end{array}$ & p value \\
\hline Sepsis & $4(16 \%)$ & $0(0 \%)$ & 0.047 \\
Shock & $10(40 \%)$ & $2(7.4 \%)$ & 0.005 \\
Anemia & $21(84 \%)$ & $16(59.3 \%)$ & 0.049 \\
Antepartum hemorrhage & $2(8 \%)$ & $1(3.7 \%)$ & 0.603 \\
Postpartum hemorrhage $(>500 \mathrm{~mL})$ & $12(48 \%)$ & $10(37 \%)$ & 0.424 \\
Postpartum hemorrhage $(>1000 \mathrm{~mL})$ & $1(4 \%)$ & $1(3.7 \%)$ & 1.000 \\
Preexisting comorbidities & $7(28 \%)$ & $5(18.5 \%)$ & 0.417 \\
HELLP syndrome & $8(32 \%)$ & $4(14.8 \%)$ & 0.142 \\
Previous surgery (including previous cesarean) & $3(12.5 \%)$ & $7(25.9 \%)$ & 0.254 \\
DIC & $0(0 \%)$ & $0(0 \%)$ & - \\
Trauma & $0(0 \%)$ & $0(0 \%)$ & - \\
Acute fatty liver & $0(0 \%)$ & $0(0 \%)$ & - \\
Radiocontrast agents & $0(0 \%)$ & $0(0 \%)$ & - \\
Diabetes mellitus & $0(0 \%)$ & $0(0 \%)$ & - \\
Nephrotoxic drugs & $0(0 \%)$ & $0(0 \%)$ & - \\
Eclampsia & $18(72 \%)$ & $21(77.8 \%)$ & 0.631 \\
Pulmonary edema & $4(16 \%)$ & $3(11.1 \%)$ & 0.698 \\
Urinary protein & $2(1-2)$ & $1(1-2)$ & 0.096 \\
\hline Valus
\end{tabular}

Values are number of patients (\% of patients within respective group) or median (IQR); HELLP, hemolysis, elevated liver enzyme, and low platelet count; DIC, disseminated intravascular coagulopathy; ${ }^{*}$ proteinuria measured by urinary dipstick method

Table 3: Comparison of clinical outcome between patients with and without AKI

\begin{tabular}{lcll}
\hline Characteristic & $\begin{array}{c}A K I \\
(n=25)\end{array}$ & $\begin{array}{l}\text { Non-AKI } \\
(n=27)\end{array}$ & $p$ value \\
\hline Duration of ICU stay (days) & $2(1-3.8)$ & $1(1-2)$ & 0.037 \\
Duration of hospital stay (days) & $13.5(8.2-17.5)$ & $9(8-10)$ & 0.021 \\
ICU mortality & $7(28 \%)$ & $1(3.7 \%)$ & 0.022
\end{tabular}

Values are number of patients (\% of patients within respective group) or median (IQR)

Comparison of outcome showed significantly longer duration of stay in the ICU and hospital, as well as greater ICU mortality for patients with AKI ( $p<0.05$; Table 3$)$.

The use of furosemide was significantly higher in patients with AKI ( 21 of $25=84 \%$ ) as compared to those without AKI (15 of $27=55.5 \%)(p=0.026)$.

Fluid balance for the first 7 days of ICU stay among patients with AKI was clinically greater [862 (-128 to 2093) $\mathrm{mL}$ ] as compared to without it [290 (-95 to 1010$) \mathrm{mL}$, but remained statistically similar $(p=0.065)$

\section{Serum NGAL-related Results}

The serum NGAL ranged from 93 to $335 \mathrm{ng} / \mathrm{mL}$. It was statistically similar for patients with or without AKI [274 (260-335) vs 262 (228-335) ng/mL, respectively; $p=0.722$ ]; as well as survivors and non-survivors [274 (237-335) vs $280(233-328) \mathrm{ng} / \mathrm{mL}$, respectively; $p=0.859]$; but significantly higher for the eclamptic vs non-eclamptic patients [275 (253-335) and 259 (178-291) ng/mL, respectively; $p=0.018]$.

Upon ROC analysis, there was no significant predictive value of serum NGAL for mortality or AKI or mortality $(p=0.725$ and 0.861 , respectively) [area under curve $(A \cup C)=0.530(95 \% \mathrm{Cl}: 0.359-0.701)$ and 0.478 (95\% Cl: 0.256-0.699), respectively]. There was, however, a significant association of serum NGAL with occurrence of eclampsia $(p=0.019)[$ AUC $=0.736$ (95\% Cl: 0.569-0.904)] (Fig. 1)

\section{ICU Survivors vs Non-survivors}

Comparison of ICU survivors vs non-survivors was also done for certain predictors (Table 4). Among the non-survivors, there was a significantly greater incidence of AKI, need for renal replacement therapy, use of diuretics, net fluid balance, sepsis, shock, pulmonary edema, intrauterine death, and minor postpartum hemorrhage ( $p<0.05$; Table 4).

However, univariate regression analysis showed significant predictive power for the presence of AKI $[p=0.022$; OR $(95 \%$ $C I)=10.1(1.1-89.4)]$, positive fluid balance $[p=0.007$; OR $(95 \%$ $\mathrm{Cl})=1.001$ (1.000-1.002)], pulmonary edema $[p=0.002 ; \mathrm{OR}$ $(95 \% \mathrm{Cl})=16.7(2.8-97.1)]$, intrauterine fetal death $[p=0.005 ;$ OR $(95 \% \mathrm{Cl})=13.7(2.2-83.9)]$, and minor postpartum hemorrhage (i.e., $>500 \mathrm{~mL})[p=0.020$; OR $(95 \% \mathrm{Cl})=13.5(1.5-120)]$.

\section{Discussion}

The present prospective cohort was used to evaluate AKI in a specific subset of critically ill obstetric patients viz., those with preeclampsia.

There are some previous data regarding AKI in preeclamptic patients but not those admitted to ICU. ${ }^{11-13}$ The incidences reported in these previous publications showed lower percentages than observed by us (15.3 and 17\%). ${ }^{11,12}$ The higher incidence noted in our cohort may be secondary to the increased presence of risk factors for AKI, such as sepsis and shock in ICU patients.

Although there are no previous data dealing with AKI in critically ill preeclamptic patients, we could, however, locate some studies focusing on mixed obstetric population admitted to ICU. ${ }^{9,14,15}$ Herein, the incidence of AKI was noted to be 
Table 4: Comparison of ICU survivors and non-survivors

\begin{tabular}{lccc}
\hline Characteristic & $\begin{array}{c}\text { Non-survivors } \\
(n=8)\end{array}$ & $\begin{array}{l}\text { Survivors } \\
(n=44)\end{array}$ & $p$ value \\
\hline Presence of AKI & $7(87.5 \%)$ & $18(40.9 \%)$ & 0.022 \\
Need for renal replacement therapy & $2(25 \%)$ & $0(0 \%)$ & 0.021 \\
Use of diuretics & $8(100 \%)$ & $28(63.6 \%)$ & 0.040 \\
Net fluid balance $(\mathrm{mL})$ & $1885(826-3246)$ & $440(-362$ to 1122$)$ & 0.001 \\
Sepsis & $4(50 \%)$ & $0(0 \%)$ & 0.000 \\
Shock & $8(100 \%)$ & $4(9.1 \%)$ & 0.000 \\
Pulmonary edema & $5(62.5 \%)$ & $4(9.1 \%)$ & 0.000 \\
Intrauterine death & $4(50 \%)$ & $3(6.8 \%)$ & 0.001 \\
Postpartum hemorrhage $(>500 \mathrm{~mL})$ & $7(87.5 \%)$ & $15(34.1 \%)$ & 0.007 \\
Postpartum hemorrhage $(>1000 \mathrm{~mL})$ & $0(0 \%)$ & $2(4.5 \%)$ & 1.000 \\
Anemia & $8(100 \%)$ & $29(65.9 \%)$ & 0.050 \\
HELLP syndrome & $3(37.5 \%)$ & $9(20.5 \%)$ & 0.293 \\
Eclampsia & $4(50 \%)$ & $35(79.5 \%)$ & 0.076 \\
Antepartum hemorrhage & $0(0 \%)$ & $3(6.8 \%)$ & 1.000 \\
Duration of ICU stay (days) & $3(1-12)$ & $1(1-2)$ & 0.076 \\
\hline
\end{tabular}

Values are number of patients (\% of patients within respective group) or median (IQR)

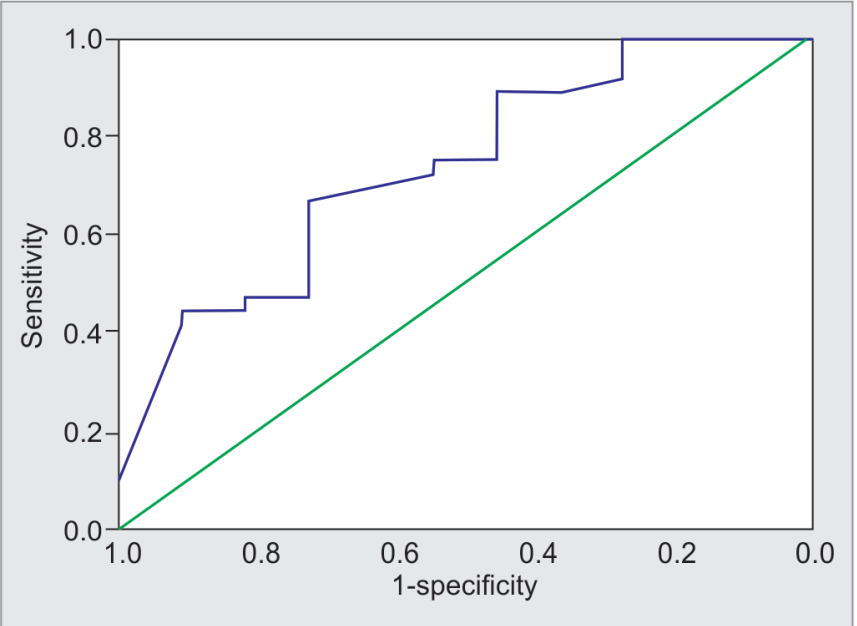

Fig. 1: ROC curve for serum NGAL and prediction of eclampsia

$5.9,33.6$, and $61 \%$, respectively. $9,14,15$ The two studies noting lower incidences ${ }^{14,15}$ than observed by us had used the risk, injury, failure, loss, end-stage (RIFLE), rather than KDIGO criteria for diagnosing AKI. The RIFLE criterion does not diagnose the mildest form of AKI (stage 1) included in KDIGO viz., increase of $\geq 0.3 \mathrm{mg} \%$ in serum creatinine over 48 hours. Excluding stage 1 of $A K I$ in our observations decreases the incidence to more similar figures of 11 of 52 (21\%).

The previous study noting a higher incidence (61\%) closer to our observation (48.1\%) had used the KDIGO criteria and thus makes for a more appropriate comparison. ${ }^{9}$ The incidence of AKI thus appears to be lesser in the critically ill preeclamptic cohort (48.1\%) as compared to one with the mixed obstetric population (61\%). Significantly lesser association of preeclampsia/eclampsia with AKI can be interpreted from secondary data of previous studies. ${ }^{9,15}$ It is possible that in preeclampsia, the severity of disease and hence associated renal complications start to decrease with placental delivery, while other causes of AKI, such as sepsis or peripartum hemorrhage, may have a longer duration of the insult. There could also be a heightened level of care from an early preoperative period in patients with preeclampsia/eclampsia, unlike with other obstetric complications that evolve unexpectedly or over prolonged durations. These reasons could contribute to lesser AKI in preeclamptic patients.

A lesser incidence of AKI in preeclamptics per se also implies that other independent factors continue to be a risk of AKI within this subset of patients. In our study sepsis, shock and anemia were significantly commoners in patients with AKI, while the severity of preeclampsia was similar when compared to those without AKI. Further studies evaluating AKI in preeclamptics vs non-preeclamptic patients as a primary objective would be beneficial.

We observed a significant association of AKI with increased mortality and duration of ICU and hospital stay, as previously wellestablished. ${ }^{8,9,15}$ The mortality rate among patients with AKI noted by us $(28 \%)$ is similar to that reported earlier in a mixed population of critically ill obstetric patients i.e., 28.3 and $33 \% .{ }^{9,15}$ The significant association of AKI with sepsis and shock is similar to previous findings when evaluating AKI in a mixed population of critically ill obstetric patients. ${ }^{9,15}$

In our study, the mildest form of AKI (stage 1) was commonest while mortality was maximum for stage 3 patients. Similar findings were noted previously in a mixed population of critically ill obstetric patients, as well as non-critically ill preeclamptic patients. ${ }^{8,9,11,12,15}$

We observed a significant predictive value of serum NGAL for eclampsia i.e., severity of preeclampsia, but not for AKI. This is similar to earlier findings of Moyake et al., although for urinary rather than serum estimates in preeclamptics. ${ }^{16}$ On the contrary, in another study by Patel et al., ${ }^{10}$ it was concluded that serum NGAL measured among preeclamptics was a successful marker of AKI. However, the authors had herein compared the levels of NGAL in preeclamptics with AKI against healthy controls. Thus, whether the higher NGAL was a result of preeclampsia or the AKI would be difficult to conclude. 
There is a plethora of publications showing the utility of NGAL for diagnosing and assessing the severity of preeclampsia. ${ }^{17-23}$ The increase in NGAL due to preeclampsia is attributed to the presence of endothelium dysfunction and systemic inflammation. ${ }^{24}$ The range of serum NGAL reported in these previous studies is very wide. This could be attributed to variations in the severity of preeclampsia ${ }^{18,20,22}$ and period of gestation, ${ }^{17,25}$ both affecting NGAL levels individually. Studies that noted levels in severe preeclampsia or eclampsia with patients in the last trimester reported levels close to our observations [237.5 (67.4-575.4) ng/mL; ${ }^{18} 407 \pm 491 \mathrm{ng} / \mathrm{mL}_{i}{ }^{22}$ and $250 \mathrm{ng} / \mathrm{mL}^{6}$ vs 274 (240-335) ng/mL, respectively].

A limitation of the present study is that it lacks a critically ill non-preeclamptic group for comparison of the findings.

Based on our observations, we conclude that AKI is common among critically ill preeclamptic patients and significantly increases the morbidity as well as mortality. AKI is significantly associated with sepsis, shock, and anemia. Serum NGAL measured at the time of ICU admission does not predict either AKI or mortality in these patients, but remains a significant predictor of eclampsia.

It is thus recommended that critically ill preeclamptic patients must be intensively monitored and managed for AKI. The presence of AKI should alert to a higher probability of death in these patients. Serum NGAL can be used as a predictor of severity of preeclampsia but not $\mathrm{AKI}$ in these patients.

\section{Author Contribution}

AT: Helped in designing the study; analyzing the data; writing/ reviewing the manuscript; approving the final submission. PY: Helped in conducting the study; writing/reviewing the manuscript; approving the final submission. RS: Helped in designing the study; writing/reviewing the manuscript; approving the final submission. SD, PS: Helped in conducting the study; writing/reviewing the manuscript; approving the final submission. DG: Helped in conducting the study; writing/reviewing the manuscript; approving the final submission.

\section{ORCID}

Asha Tyagi @ https://orcid.org/0000-0001-5331-6832

Pooja Yadav (1) https://orcid.org/0000-0002-2519-9023

Rashmi Salhotra (1) https://orcid.org/0000-0002-2078-1583

Shukla Das 이 https://orcid.org/0000-0002-8117-2423

Praveen K Singh (1) https://orcid.org/0000-0003-2429-7913

Devansh Garg @ https://orcid.org/0000-0002-3728-0398

\section{References}

1. Hutcheon JA, Lisonkova S, Joseph KS. Epidemiology of preeclampsia and the other hypertensive disorders of pregnancy. Best Pract Res Clin Obstet Gynaecol 2011;25(4):391-403. DOI: 10.1016/j. bpobgyn.2011.01.006.

2. Hypertensive disorders in pregnancy. In: Dutta DC, Konar H, editors. Textbook of obstetrics. 8th ed. New Delhi: Jaypee; 2015. p. 256-267.

3. American College of Obstetricians and Gynecologists. ACOG Practice Bulletin No. 202: gestational hypertension and preeclampsia. Obstet Gynecol 2019;133(1):e1-e25. DOI: 10.1097/AOG.0000000000003018.

4. Bateman BT, Polley LS. Hypertensive disorders. In: Chestnut DH, Wong CA, Tsen LC, Ngan Kee WD, Beilin Y, Mhyre JM, editors. Chestnut's obstetric anaesthesia. 5th ed. Philadelphia: Elsevier; 2014. p. 826-850.

5. Codsi E, Garovic VD, Gonzalez-Suarez ML, Milic N, Borowski KS, Rose $\mathrm{CH}$, et al. Longitudinal characterization of renal proximal tubular markers in normotensive and preeclamptic pregnancies. Am J Physiol Regul Integr Comp Physiol 2017;312(5):R773-R778.

6. Simonazzi G, Capelli I, Curti A, Comai G, Rizzo N, La Manna G. Serum and urinary neutrophil gelatinase-associated lipocalin monitoring in normal pregnancy versus pregnancies complicated by preeclampsia. In Vivo 2015;29(1):117-121.

7. Moran P, Baylis PH, Lindheimer MD, Davison JM. Glomerular ultrafiltration in normal and preeclamptic pregnancy. J Am Soc Nephrol 2003;14(3):648-652. DOI: 10.1097/01.asn.0000051724.66235.e0.

8. Kidney disease: improving global outcome. Available at: https://www. kdigo.org [Accessed on August 15, 2020].

9. Tyagi A, Luthra A, Kumar M, Das S. Epidemiology of acute kidney injury and the role of urinary [TIMP-2].[IGFBP7]: a prospective cohort study in critically ill obstetric patients. Int J Obstet Anesth 2018;36:77-84. DOI: 10.1016/j.ijoa.2018.08.002.

10. Patel M, Sachan R, Gangwar R, Sachan P, Natu S. Correlation of serum neutrophil gelatinase-associated lipocalin with acute kidney injury in hypertensive disorders of pregnancy. Int J Nephrol Renovasc Dis 2013;6:181-186. DOI: 10.2147/IJNRD.S45523.

11. Conti-Ramsden FI, Nathan HL, De Greeff A, Hall DR, Seed PT, Chappell LC, et al. Pregnancy-related acute kidney injury in preeclampsia: risk factors and renal outcomes. Hypertension 2019;74(5):1144-1151. DOI: 10.1161/HYPERTENSIONAHA.119.13089.

12. Samejima T, Yamashita T, Takeda Y, Adachi T. Low antithrombin levels accompanied by high urine protein/creatinine ratios are predictive of acute kidney injury among CS patients with preeclampsia. J Matern Fetal Neonatal Med 2021;34(10):1550-1556. DOI: 10.1080/14767058.2019.1639662.

13. Tangren JS, Wan Md Adnan WAH, Powe CE, Ecker J, Bramham $\mathrm{K}$, Hladunewich MA, et al. Risk of preeclampsia and pregnancy complications in women with a history of acute kidney injury. Hypertension 2018;72(2):451-459. DOI: 10.1161/ HYPERTENSIONAHA.118.11161.

14. Kamal EM, Behery MM, Sayed GA, Abdulatif HK. RIFLE classification and mortality in obstetric patients admitted to the intensive care unit with acute kidney injury: a 3-year prospective study. Reprod Sci 2014;21(10):1281-1287. DOI: 10.1177/1933719114525277.

15. Bentata Y, Housni B, Mimouni A, Azzouzi A, Abouqal R. Acute kidney injury related to pregnancy in developing countries: etiology and risk factors in an intensive care unit. J Nephrol 2012;25(5):764-775. DOI: 10.5301/jn.5000058.

16. Moyake N, Buchmann E, Crowther NJ. Neutrophil gelatinaseassociated lipocalin as a diagnostic marker of acute kidney injury in pre-eclampsia. J Obstet Gynaecol Res 2016;42(11):1483-1488. DOI: 10.1111/jog.13088.

17. D'Anna R, Baviera G, Giordano D, Todarello G, Russo S, Recupero S, et al. Neutrophil gelatinase-associated lipocalin serum evaluation through normal pregnancy and in pregnancies complicated by preeclampsia. Acta Obstet Gynecol Scand 2010;89(2):275-278. DOI: 10.3109/00016340903443676.

18. Kim SM, Park JS, Norwitz ER, Jung HJ, Kim BJ, Park CW, et al. Circulating levels of neutrophil gelatinase-associated lipocalin (NGAL) correlate with the presence and severity of preeclampsia. Reprod Sci 2013;20(9):1083-1089. DOI: 10.1177/1933719113477480.

19. Artunc-Ulkumen B, Guvenc Y, Goker A, Gozukara C. Relationship of neutrophil gelatinase-associated lipocalin (NGAL) and procalcitonin levels with the presence and severity of the preeclampsia. J Matern Fetal Neonatal Med 2015;28(16):1895-1900. DOI: 10.3109/14767058.2014.972926.

20. Sachan R, Patel ML, Gaurav A, Gangwar R, Sachan P. Correlation of serum neutrophil gelatinase associated lipocalin with disease severity in hypertensive disorders of pregnancy. Adv Biomed Res 2014;3:223. DOI: 10.4103/2277-9175.145690.

21. Yuksel S, Ozyurek SE, Acar DK, Ozdemir C, Guler S, Kiyak H, et al. Urinary neutrophil gelatinase-associated lipocalin is associated with preeclampsia in a cohort of Turkish women. Hypertens Pregnancy 2019;38(3):157-162. DOI: 10.1080/10641955.2019.1621887. 
22. Cui L, Shu C, Liu Z, Tong W, Cui M, Wei C, et al. Serum protein marker panel for predicting preeclampsia. Pregnancy Hypertens 2018;14:279-285. DOI: 10.1016/j.preghy.2018.01.009.

23. Scazzochio E, Munmany M, Garcia L, Meler E, Crispi F, Gratacos E, et al. Prognostic role of maternal neutrophil gelatinase-associated lipocalin in women with severe early-onset preeclampsia. Fetal Diagn Ther 2014;35:127-132. DOI: 10.1159/000356499.
24. Huppertz B. Placental origins of preeclampsia: challenging the current hypothesis. Hypertension 2008;51(4):970-975. DOI: 10.1161/ HYPERTENSIONAHA.107.107607.

25. D'Anna R, Baviera G, Giordano D, Todarello G, Corrado F, Buemi M. Second trimester neutrophil gelatinase associated lipocalin as a potential prediagnostic marker of preeclampsia. Acta Obstet Gynecol Scand 2008;87(12):1370-1373. DOI: 10.1080/00016340802464463. 